\title{
Mujeres jóvenes con infección por VIH adquirida por transmisión vertical. Expectativas de tener hijos no infectados
}

\author{
Julia Villarroel, Ana M. Álvarez, Francisco Salvador, Ana Chávez, Elba Wu, y Verónica Contardo
}

Sociedad Chilena de Pediatría Comité Nacional de SIDA Pediátrico (JV, AMA, ACH, EW). Universidad de Antofagasta Facultad de Medicina y Odontología (FS).

Universidad de Chile.

Facultad de Medicina Departamento de Pediatría Norte (VC)

Fuentes de financiamiento: Propias. Los autores declaran no tener conflictos de interés.

Recibido: 28 de noviembre de 2015 Aceptado: 17 de octubre de 2016

Correspondencia a Julia Villarroel Barrera julia.villarroel@vtr.ne

\section{Young women with HIV infection acquired by vertical transmission. Expectations of having uninfected children}

Introduction: Pediatric antiretroviral therapy (ART), changed the prognosis of the disease, allowing young women infected by vertical transmission (TV) to be pregnant without risk for their fetus of acquiring this infection. Aim: To describe the clinical-immune status in pregnant women that acquired HV by vertical transmission, treatments received, monitoring of pregnancy and newborn characteristics. Material and Methods: A protocol was performed, evaluating clinical and immunological parameters during pregnancy, ART used, protocol preventing vertical transmission (PPTV), and follow up of children to 18 months of age. Results: Of 358 HIV-positive patients vertically infected, five women became pregnant, between 14 and 24 years old. Pregnancies were controlled in clinical/immune-stage N2 C3. They had received two to five therapies. Full PPTV was performed in all binomials. Pre-natal undetectable viral loads ranged from 4,700 ARN copies $/ \mathrm{mL}$. Five living children were born by Caesarean section, four of them with 37 weeks of completed gestation and one of them with 34 weeks of gestation. All received zidovudine (AZT) for 6 weeks. CD4 at 72 hours of life ranged from $48 \%$ to $74.6 \%$. All children were born uninfected with HIV. Only two had mild anemia. Conclusions: Expectations of HIV mothers vertically infected to have healthy children are similar to those infected by horizontal transmission, using PPTV.

Key words: pediatric HIV, vertical transmission, pregnant young woman.

Palabras clave: VIH pediátrico, transmisión vertical, mujeres jóvenes embarazadas.

\section{Introducción}

$\mathrm{H}$ ace 26 años se describieron en Chile, los primeros casos de niños infectados por VIH que adquirieran la infección por transmisión vertical (TV), algunos de los cuales no sobrevivieron más allá de la infancia. Sin embargo, con la introducción de la terapia anti-retroviral de alta eficiencia (TARV), el pronóstico de la enfermedad cambió, así como la sobrevida ${ }^{1}$, por lo que tenemos ahora mujeres adolescentes y adultas jóvenes, en edad de embarazarse y eventualmente, una segunda generación de pacientes infectados con VIH, en quienes la forma de transmisión también sea vertical.

Desde el año 1998 se están reportando en el mundo ${ }^{2,3}$, embarazos en mujeres que adquirieran la infección por VIH en forma vertical. Nuestro país no está exento de esta problemática; a junio del año 2015 se han estudiado 2.590 binomios madre-hijo, diagnosticándose infección por VIH en 358 niños. Dentro de estos últimos, cinco mujeres se embarazaron y recibieron protocolo de prevención de la transmisión vertical (PPTV) completo según la normativa vigente $^{4}$

El Comité de VIH/SIDA Pediátrico, de la Sociedad Chilena de Pediatría, estimó pertinente y de interés presentar estos casos, por las implicancias a futuro de este tema.

\section{Objetivos}

Conocer las características clínicas e inmunológicas de las mujeres portadoras del VIH que se embarazaron, la(s) TARV recibida (s) previamente, su condición al momento del embarazo y el seguimiento de los recién nacidos (RN) de esta segunda generación.

\section{Pacientes y Métodos}

A cada una de las mujeres embarazadas se les administró el PPTV del VIH. Se evaluaron las etapas clínicoinmunológicas al momento del embarazo, se registraron las TARV recibidas previamente, las genotipificaciones realizadas durante el embarazo y los anti-retrovirales (ARV) usados. En los RN se completó el PPTV del VIH y se inició el proceso diagnóstico de la infección ${ }^{4}$. Estos niños fueron seguidos por el Comité hasta los 18 meses de vida, edad en que se estableció definitivamente la existencia o no existencia de infección por VIH en ellos.

El análisis e interpretación de sensibilidad genotípica 


\begin{tabular}{|c|c|c|c|c|c|c|c|}
\hline \multirow[t]{2}{*}{ Caso $\mathrm{N}^{\circ}$} & \multicolumn{4}{|c|}{ Etapificación y carga viral } & \multirow{2}{*}{$\begin{array}{l}\text { Edad al parto } \\
\text { (años) }\end{array}$} & \multirow{2}{*}{$\begin{array}{c}\text { Fecha del } \\
\text { parto }\end{array}$} & \multirow{2}{*}{$\begin{array}{l}\text { Hospital de } \\
\text { atención }\end{array}$} \\
\hline & $\begin{array}{l}\text { Fecha de } \\
\text { CV y CD4 }\end{array}$ & Etapa clínica & $\begin{array}{c}\text { CD4 } \\
\left(\mathrm{N} / \mathrm{mm}^{3}-\%\right)\end{array}$ & $\begin{array}{c}\text { CV } \\
\text { (copias ARN/ml-log) }\end{array}$ & & & \\
\hline 1 & $27 / 11 / 12$ & C3 & $168 / 16$ & $94-1,97$ & 24 & $09 / 12 / 12$ & SJD \\
\hline 2 & $14 / 05 / 13$ & N2 & $376 / 20$ & $2.760-3,44$ & 21 & $23 / 07 / 13$ & EGC \\
\hline 3 & $14 / 02 / 13$ & N3 & $190 / 10,1$ & Indetectable & 18 & $16 / 04 / 13$ & SJD \\
\hline 4 & $02 / 12 / 13$ & N2 & $284 / 19,8$ & $23-1,36$ & 18 & $30 / 12 / 13$ & EGC \\
\hline 5 & 03/04/07 & $\mathrm{C} 2$ & $236 / 24$ & $4.700-3,67$ & 14 & 26/09/07 & EGC \\
\hline
\end{tabular}

a los medicamentos se realizó de acuerdo al programa online de la Universidad de Stanford, en busca de una correlación de éstas, con el resultado clínico final ${ }^{5}$.

\section{Resultados}

De los 358 niños infectados con VIH por TV, luego cinco mujeres se embarazaron. Sus edades al momento de iniciar la gestación fluctuaron entre 14 y 24 años (promedio19 años), sólo una era menor de edad. Todas eran solteras y tenían residencia en la Región Metropolitana.

Sus embarazos fueron controlados por los equipos de Alto Riesgo Obstétrico y VIH/SIDA de Adultos; en ninguna de ellas se detectó otras enfermedades de transmisión sexual y no acusaban abuso de alcohol o drogas. Una de ellas tuvo síntomas de parto prematuro, pero no se detectó otra patología obstétrica.

Según la clasificación de VIH en niños, se encontraban en etapa clínico-inmunológica al embarazo entre N2 y C3 (Tabla 1) ${ }^{6}$.

Todos los partos fueron resueltos vía cesárea, en cuatro fue indicación electiva y en uno cesárea de urgencia. En dos casos las parejas sexuales desconocían la condición de infectadas de las mujeres embarazadas.

En la Tabla 2, se muestran las genotipificaciones efectuadas a estas mujeres embarazadas. Sólo se pudo obtener genotipificación en tres de las cinco pacientes; en una no se pudo realizar, porque había abandonado la TARV durante cinco años y la otra paciente estaba con carga viral (CV) indetectable desde el año 2004. Estas mujeres habían recibido entre dos y cinco esquemas de TARV previos; en dos de las cinco, la terapia inicial fue biterapia (dos análogos de nucleósidos, incluyendo zidovudina (AZT)). Sólo uno de los casos (caso 1) mostraba resistencia intermedia a AZT en la genotipificación realizada durante la gestación. Las genotipificaciones de las tres pacientes en que se pudo realizar, mostraban resistencia a los inhibidores de transcriptasa reversa no nucleósidos
(ITRNN) nevirapina (NVP) y efavirenz (EFV), ARVs muy usados en las TARV iniciales en niños.

Durante el embarazo, tres de ellas recibieron abacavir/ lamivudina (ABC/3TC) más lopinavir/ritonavir (LPV/ $\mathrm{rtv}$ ), una darunavir/ritonavir (DRV/rtv) más (ABC/3TC). En el caso $\mathrm{N}^{\circ} 1$ se necesitó agregar raltegravir (RAL) en las últimas semanas de embarazo, por aumento de la CV con lo cual, su CV bajó a 94 copias ARN/mL (log 1,97), previo al parto, siendo la última genotipificación negativa para resistencia a AZT. El caso $\mathrm{N}^{\circ} 5$, quien se diagnosticó en etapa $\mathrm{C} 2$, inició TARV con AZT + ddI con mala adherencia, terapia que se mantuvo durante ocho años, luego desapareció de controles médicos y abandonó la terapia, por más de cinco años, hasta que a los 14 años se embarazara. La gestación fue controlada en un hospital donde, por abandono de la TARV durante largos años, no se efectuó genotipificación del VIH, iniciándose AZT$3 \mathrm{TC}+$ saquinavir + ritonavir, luego AZT durante el parto y en el recién nacido, quien lo recibió por seis semanas.

Se efectuó PPTV completo en todos los binomios madre-hijo, por lo que no fue necesario el uso de NVP en la prevención. Las $\mathrm{CV}$ que presentaban previas al parto, fluctuaron entre indetectable y 4.700 copias ARN/ $\mathrm{mL}(\log 3,67)$.

Nacieron cinco niños vivos, cuatro de término adecuados a la edad gestacional y uno prematuro con 34 semanas de gestación, quien presentó un síndrome de distress respiratorio que requirió aporte de oxígeno, con buena evolución posterior. Los cinco $\mathrm{RN}$ recibieron profilaxis solamente con AZT. Los estudios etiológicos de VIH efectuados en estos niños durante el seguimiento, descartaron definitivamente la infección vertical por VIH.

Los porcentajes de CD4 realizados a las 48 a $72 \mathrm{~h}$ de vida fluctuaron entre 48 y 74,6\%, con recuentos entre 1.770 y 2.827 céls $/ \mathrm{mm}^{3}$. Dos niños presentaron anemia hipocroma leve, que se recuperó. Ninguno tuvo acidosis láctica, neutropenia, compromiso hepático o infección por citomegalovirus. 
VIH / SIDA

\begin{tabular}{|c|c|c|c|c|}
\hline Caso $\mathrm{N}^{\circ}$ & $\mathrm{N}^{\circ}$ genotipificación & Fecha de genotipificación & Resultados & Mutaciones \\
\hline \multirow[t]{2}{*}{1} & 1 & $11-05-12$ & $\begin{array}{l}\text { INTR: Resistencia intermedia: AZT, d4T, ddl, TDF, ABC } \\
\text { Sensible: } 3 T C \\
\text { INNTR: resistente a EFV, NVP } \\
\text { IP: resistencia: atazanavir, fosamprenavir, lopinavir, } \\
\text { nelfinavir, saquinavir } \\
\text { Sensible: darunavir, tripanavir }\end{array}$ & $\begin{array}{c}\text { INTR: M41L, L210W, T215C } \\
\text { INNRT: L100I, K103N } \\
\text { IP: I54V, V82A, L90M, L10I, A71V, K20R, } \\
\text { M36I, I62V, L63P, V77I, 193L }\end{array}$ \\
\hline & 2 & $20-08-12$ & $\begin{array}{l}\text { INTR: } \sin \text { resistencia } \\
\text { INNTR: sin resistencia } \\
\text { IP: sin resistencia }\end{array}$ & L10I, A71T \\
\hline 2 & $\begin{array}{l}\text { Sin genotipificación, } \\
\text { indetectable desde } 2004\end{array}$ & & & \\
\hline \multirow[t]{3}{*}{3} & 1 & 05-10-05 & $\begin{array}{l}\text { INRT: } \sin \text { resistencia } \\
\text { INNTR: Resistencia: EFV, NVP } \\
\text { IP: sin resistencia }\end{array}$ & $\begin{array}{l}\text { K101Q } \\
\text { K103N } \\
\text { P225H }\end{array}$ \\
\hline & 2 & $25-10-07$ & $\begin{array}{l}\text { INRT: } \sin \text { resistencia } \\
\text { INNTR: Resistencia: EFV, NVP } \\
\text { IP: sin resistencia }\end{array}$ & $\begin{array}{l}\text { K101Q } \\
\text { K103N } \\
\text { P225H }\end{array}$ \\
\hline & 3 & 18-08-09 & $\begin{array}{l}\text { INRT: sin resistencia } \\
\text { INNTR: Resistencia: EFV, NVP. } \\
\text { IP: sin resistencia }\end{array}$ & $\begin{array}{l}\text { K101Q } \\
\text { K103N } \\
\text { P225H }\end{array}$ \\
\hline 4 & 1 & $26-03-12$ & $\begin{array}{l}\text { INTR: sin resistencia } \\
\text { INNTR: Resistencia EFV,NVP } \\
\text { IP: sin resistencia }\end{array}$ & $\begin{array}{c}\text { M41L, Y188L, T215D } \\
\text { L10V }\end{array}$ \\
\hline 5 & $\begin{array}{l}\text { Sin genotipificación, } \\
\text { Por abandono de TARV } \\
\text { hasta el embarazo }\end{array}$ & & & \\
\hline
\end{tabular}

\section{Discusión y conclusiones}

Poco se conoce sobre los embarazos en mujeres que adquirieran la infección por VIH de manera vertical; una serie de factores hacen que estas mujeres tengan una gestación con mayor riesgo para sus hijos(as), tales como la exposición intrauterina del cerebro al VIH, los potenciales efectos teratogénicos de la TARV, la desregulación del sistema inmune materno, su disfunción vascular y alteraciones del sistema cardiovascular y un alto riesgo de resistencia viral por las múltiples terapias utilizadas con anterioridad $^{6}$. Todo ello puede hacer que los embarazos y partos en esta población sean diferentes al de mujeres no infectadas, e incluso, diferentes al de mujeres infectadas por transmisión horizontal. En los casos estudiados por nosotros, ninguna de las pacientes embarazadas había sido expuesta a ARVs en su vida in utero, debido a que aún no estaba implementado el PPTV en los años en que ellas nacieron.

Un interesante estudio retrospectivo comparó la gestación de 20 mujeres que habían sido infectadas de manera vertical versus 80 mujeres infectadas por transmisión horizontal, entre los años 2007 y 2012. Hubo similitud entre el recuento de CD4, la prevalencia de SIDA y el uso de TARV al inicio de la atención; sin embargo, las mujeres infectadas por TV eran más jóvenes, presentaban mayor resistencia genotípica (40 versus $12 \%$ ) y tenían mayores $\mathrm{CV}(65 \%$ versus $26 \%)$, pero no presentaron diferencias en la edad gestacional al momento del parto ni en la TV del VIH al RN; tampoco tuvieron complicaciones obstétricas, ni en los RN se detectó alguna patología 7 .

En otras latitudes se han estudiado los embarazos de mujeres con VIH adquirido por TV y por otras vías, encontrando que las primeras tienen menor recuento de 
$\mathrm{CD} 4$ y mayor $\mathrm{CV}$, mayor resistencia viral, mayor tasa de cesáreas pero similar tasa de complicaciones maternoneonatales, incluyendo parto prematuro ${ }^{8,9}$. En los casos estudiados por nosotros, la edad promedio de las mujeres embarazadas fue de 19 años lo que concuerda con trabajos extranjeros ${ }^{9} \mathrm{y}$, en uno de nuestros casos, el embarazo ocurrió en una adolescente de 14 años.

Otros estudios han tenido resultados opuestos, describiéndose una mayor cantidad de partos prematuros en el grupo de mujeres gestantes infectadas en forma perinatal, $(31 \%)$ versus la población normal $(13 \text { a } 18 \%)^{10,11}$. No está aclarada la causa, pero se atribuye a factores como el uso de TARV en el embarazo, el abusar de drogas y estar con una enfermedad por VIH más avanzada. Tampoco es clara la asociación con las combinaciones de TARV, siendo posible un aumento del riesgo en mujeres que reciben inhibidores de proteasas (IP). En nuestra pequeña serie se produjo sólo un parto prematuro.

En el aspecto psico-social, las diferentes series muestran alta prevalencia de abuso de sustancias, alteraciones mentales, desarrollo neurocognitivo muy inferior a la población normal de la misma edad, manifestaciones neuro-psiquiátricas tales como depresión, estados de ansiedad, impulsividad, con una frecuencia de hasta $60 \%$, lo que puede provocar problemas serios, no sólo en el embarazo, sino también en el post parto, con tremendas repercusiones para el $\mathrm{RN}^{12-15}$. Se ha demostrado que el desarrollo neurocognitivo y las alteraciones mentales influyen directamente en la adherencia de estas pacientes a la TARV ${ }^{16-19}$. Nosotros podemos decir en los casos estudiados, que no hubo abuso de alcohol o drogas; no obstante, no disponemos de estudio neuro-psiquiátrico y cognitivo de cada una de ellas.

El ocultar el diagnóstico de infección VIH a su pareja, descrito en trabajos extranjeros hasta en $40 \%$ de los casos, al igual que tener relaciones sexuales sin protección ${ }^{20,21}$ es otro aspecto a considerar; en dos de los cinco casos descritos por nosotros, la pareja desconocía la condición de infectada por VIH de la mujer.

Otros estudios también muestran que las complicaciones obstétricas más frecuentes fueron la pre-eclampsia $(23 \%)$ y rotura prematura de membrana $(31 \%)^{10-12}$. El caso de parto prematuro de nuestra serie, ocurrió por rotura prematura de membranas; los otros casos no tuvieron complicaciones obstétricas.

También se ha descrito que estas mujeres poseen virus más resistentes a los ARVs, probablemente por haber sido sometidas a múltiples TARV y con fallas en la adherencia $^{22}$, especialmente durante la adolescencia, situación que corroboramos. Estudios extranjeros muestran que los RN infectados con VIH por TV de madres con infección por VIH multi-resistentes, debieron recibir profilaxis con tres ARVs ${ }^{22-24}$.

Un punto interesante es que los cinco RN controlados por nosotros, recibieron profilaxis solamente con AZT, a pesar que todas las madres habían recibido inicialmente TARV que incluían AZT, en esquemas bi-asociados, por lo que la probabilidad de resistencia en ellas a este ARV era alta. Sin embargo, los estudios de genotipificación realizados a las madres mostraron resistencia intermedia a AZT solamente en un caso (caso 1). Esto se podría atribuir a la reaparición de cepas salvajes sensibles a AZT por el largo período sin ese ARV.

En nuestros casos, porque sus TARV iniciales contemplaron AZT, incluso como biterapia, era posible que los $\mathrm{RN}$, al quedar sólo con AZT como ARV profiláctico, se hubiesen infectado con VIH en el periparto; sin embargo, ello no fue así y los estudios etiológicos de VIH seguidos hasta los 18 meses de edad, demostraron que ninguno de los niños adquirió la infección. Esto se podría atribuir a que el AZT es efectivo incluso en cepas resistentes como lo demostraron los primeros estudios con el PPATG-076 que usaba sólo AZT como ARV ${ }^{25-28}$.

Por el uso de inhibidores de la transcriptasa reversa no nucleósidos en las terapias iniciales de nuestros casos, la resistencia a NVP y EFV-mutación K103N en dos de los casos y mutación Y188L en el otro; uno de los casos con mutación K103N tenía además una mutación L100I- estaba presente en los tres casos en que se efectuó genotipificación, pero ninguno de ellos necesitó el uso de NVP en la prevención de la TV del VIH por haber tenido un PPTV del VIH completo. Y188L, es una mutación no polimórfica que causa resistencia de alto nivel $(>50$ veces menor sensibilidad) para NVP y EFV. K103N, es una mutación no polimórfica que causa resistencia de alto nivel a NVP (alrededor de 50 veces susceptibilidad reducida) y EFV (alrededor de 20 veces susceptibilidad reducida). Y la mutación L100I es una mutación no polimórfica, que habitualmente se presenta en combinación con K103N. En esta configuración hace que la resistencia sea de alto nivel a NVP y EFV ( $>50$ veces menor susceptibilidad).

Otro aspecto poco estudiado, es qué sucede con el crecimiento de los hijos en uno y otro caso. En relación a esto, hay un estudio reciente ${ }^{29}$ efectuado en Estados Unidos de América que compara ambos grupos apreciándose un menor crecimiento durante el primer año de vida en los hijos de madres con infección por VIH de transmisión vertical; las causas de por qué se produce esto no están claras. En nuestra pequeña serie hemos podido observar un crecimiento adecuado de los cinco niños.

Los diferentes estudios realizados en este universo de pacientes muestran gran variabilidad en los resultados, lo cual evidencia la importancia de conocer cuál es el riesgo para los $\mathrm{RN}$ de mujeres con infección por VIH adquirida en el período perinatal en nuestra población, ya que todo hace predecir que la incidencia de mujeres embarazadas en esta población, va a ir rápidamente en aumento en la próxima década. 


\section{Resumen}

Introducción: La terapia anti-retroviral en pediatría (TARV), cambió el pronóstico de la enfermedad, permitiendo embarazarse a mujeres jóvenes infectadas por transmisión vertical (TV). Objetivos: Conocer las características clínico-inmunológicas de las mujeres embarazadas, tratamientos recibidos, condición al embarazo y seguimiento de sus recién nacidos. Material y Método: Se efectuó un protocolo, evaluando etapas clínico-inmunológicas en el embarazo, TARV usadas, protocolo de prevención de transmisión vertical (PPTV) y seguimiento de los niños hasta 18 meses. Resultados: De 358 pacientes con infección por VIH adquirida por TV, cinco mujeres se embarazaron, con edades entre 14 a 24 años, embarazos que fueron controlados por el equipo de salud, encontrándose en etapa clínico-inmunológica N2 a C3. Habían recibido dos a cinco esquemas de TARV. Se efectuó PPTV completo en todos los binomios. Las cargas virales previas al parto fluctuaron entre indetectable y 4.700 copias ARN/ml. Nacieron por cesárea cinco niños vivos, cuatro de término y uno con 34 semanas de gestación. Todos recibieron zidovudina (AZT) durante seis semanas. Los CD4 a las $72 \mathrm{~h}$ de vida fluctuaron entre 48 y 74,6\%. Ninguno de los niños adquirió la infección por VIH en forma vertical. Sólo dos presentaron anemia leve. Conclusiones: Las expectativas de madres con infección por VIH de adquisición vertical de tener hijos sanos son semejantes a las infectadas por transmisión horizontal, al usar PPTV.

\section{Referencias bibliográficas}

1.- Gortmaker S, Hughes M, Cervia J. Effect of combination therapy including protease inhibitors on mortality among children and adolescents infected with HIV-1. N Engl J Med 2001; 345: 1522-8

2.- Crane S, Sullivan M, Feingold M, Kaufman G Successful pregnancy in an adolescent with perinatally acquired human inmunodeficiency virus. Obstet Gynecol 1998; 92: 711.

3.- Zorrilla C, Febo I, Ortiz I, Orengo J, Miranda S, Rodríguez A, et al. Pregnancy in the perinatally HIV infected adolescents and young adults in Puerto Rico. MMWR Morbid Mortal Wkly Rep 2002; 52: 149-51

4.- Norma Conjunta de Prevención de la Transmisión Vertical del VIH y la Sífilis. Rev Chilena Infectol 2013; 30: 259-302.

5.- HIVdb Program; Genotypic resistance interpretation algorithm, Stanford University, http://sierra2.stanford.edu/sierra/servlet/ JSierra?action=mutationsInput; abril de 2015 .

6.- CDC: 1994 Revised classification system for human immunodeficiency virus infection in children less than 13 years of age. MMWR Morbid Mortal Wkly Rep 1994; 43 (RR-12): 1-10.

7.- $\quad$ Fair C, Wiener L, Zadeh S, Albright J, Mellins C, Mancilla M, et al. Reproductive health decision-making in perinatally HIV-infected adolescents and young adults. Matern Child Health J 2013; 5: 797-808.

8.- Badell M, Kachikis A, Haddad L, Nguyen M, Lindsay M. Comparison of pregnancies between perinatally and sexually HIV-infected women: An observational study at an urban hospital. Infect Dis Obstet Gynecol 2013 : $1-6$

9.- Munjol L, Dobroszycki J, Fakiglu E, Rosemberg M, Wisnia A, Kats M, et al.
Impact of HIV-1 infection and pregnacy on maternal health: comparison between perinatally and behaviorally infected young women. Adolesc Health Med Ther 2013; 4: 51-8.

10.- Phillips UK, Rosemberg M, Dobroszycki J, Katz M, Sansary J, Golatt M, et al. Pregnancy in women with perinatally acquired HIV -infection: outcomes and challenges. AIDS Care 2011; 9: 1076-82.

11.- Jolly M, Sebire N, Harris J, Robinson S, Regan L. Obstetric risks of pregnancy in women less than 18 years old. Obstet Gynecol 2000; 96: 962-6.

12.- Beckerman K, Giovanniello A, Wright R. Retrospective cohort comparing pregnancy outcome among perinatally infected women compared to sexually infected. Proc IDSA Annual Meeting. October, 2011: 20-3.

13.- Williams S, Keane-Tarchichi M, Bettica L, Dieudonne A, Bardeguez A. Pregnancy outcomes in young women with perinatally acquired human immunodeficiency virus- 1 . Am J Obstet Gynecol. 2009; 200: 149e1-5.

14.- Mellins C, Brackis-Cott E, Leu C. Rates and types of psychiatric disorders in perinatally human immunodeficiency virus-infected youth and seroreverters. J Child Psychol Psychiatry 2009; 50: 1131-8.

15.- Badell M L, Lindsay M. Thirty years later: pregnancies in females perinatally infected with human immunodeficiency virus-1. Aids Res Treat. 2012: Article ID 418630, 6 pages doi:10.1155/2012/418630.

16.- Mellins C, Tassiopoulos K, Malee K, Moscicki A, Patton D, Smith R, et al. Behavioral health risks in perinatally HIVexposed youth: co-occurrence of sexual and drug use behavior, mental health problems, and non adherence to antiretroviral treatment. Aids Patient Care STDS. 2011; 25: 413-22.
17.- Millery M, Vázquez S, Walther V, Humphrey N, Schlecht J, Van Devanter N. Pregnancies in perinatally HIV infected young women and implications for care and service programs. J Assoc Nurses AIDS Care, 2011; 23: 41-50.

18.- Jeremy R, Kim S, Nozyce M. Neuropsychological functioning and viral load in stable antiretroviral therapyexperienced HIV-infected children. Pediatrics 2005; 115: 380-7.

19.- Nozyce M, Lee S Wisnia A. A behavioral and cognitive profile of clinically stable HIV-infected children. Pediatrics 2006; 117: 763-70.

20.- Malle K, Williams P, Montepiedra G. The role of cognitive functioning in medication adherence of children and adolescents with HIV infection. J Pediatric Psycol 2009; 34 164-75.

21.- Michaud P, Suris J, Thomas R, Kahlert C, Rudin C, Cheseaux J, et al. To say or not to say: A qualitative study on the disclosure of their condition by human immunodeficiency viruspositive adolescent. J Adolesc Health 2009; 44 356-62.

22.- Karchava M, Pulver W, Smith L, Philpott S, Sullivan T, Wethers J, el al. Prevalence of drug-resistance mutations and no subtype B strains among HIV-infected infants from New York State. J Acquir Immune Defic Syndr 2006; 42: 614-9.

23.- Dryden-Peterson S, Jayeoba O, Hughes M D. Highly active antiretroviral therapy versus zidovudine for prevention of mother-to-child transmission in a programmatic setting, Botswana. J Acquir Immune Defic Syndr 2011; 58: 353-7.

24.- Afani S A, Gallardo O. Resistencia a la terapia antiretroviral en la infección por virus de inmunodeficiencia humana. Rev Chil Infectol 2011; 28: 461-9. 
25.- Delaugerre C, Chaix M, Blanche S, Warszawski J, Cornet D, Dollfus C, et al. Perinatal acquisition of drug-resistant HIVinfection: mechanisms and long-term outcome. Retrovirol 2009; 6: 85-98.

26.- Newell M L. Prevention of mother-to-child transmission of HIV: challenges for the current decade. Bull World Health Org 2001; 79: 1138-44.
27.- Coetzee D, Hilderbrand K, Boulle A, Draper B, Abdullah F, Goemaere E. Effectiveness of the first district-wide programme for the prevention of mother-to-child transmission of HIV in South Africa. Bull World Health Org 2005; 83: 489-94

28.- Calitri C, Gabiano C, Galli L, Chiappini E, Giaquinto C, Buffolano W, et al. The second generation of HIV-1 vertically exposed infants: a case series from the Italian register for paediatric HIV infection. BMC Infect Dis 2014; 14: 277-85.

29.- Jao J, Agwu A, Mhango G, Kim A, Park A, Posada R, et al. Growth patterns in the first year of life differ in infants born to perinatally vs nonperinatally HIV-infected women. AIDS 2015; 1: 111-6. 